\title{
Cardiac Output Measurement by the Injection Method Without Arterial Sampling
}

Y. van der Feer, Ph.D., J. H. Douma, M.Sc., and W. Klip, M.Sc., M.D. Utrecht, Netherlands

\section{INTRODUCTION}

In the original injection method, as introduced by Stewart, ${ }^{8}$ in 1921 , an indicator is injected intravenously, and the arterial concentration of this indicator measured as a function of time by taking samples of blood every 2 seconds or so for about 1 minute. Cardiac output is then calculated by dividing the known amount of indicator by the integral of the primary part of the concentrationtime curve, that is, that part of the curve belonging to the indicator passing the sampling site for the first time.

If a radioactive indicator, emitting $\gamma$-rays is used, the concentration-time curve can be measured outside the body by recording with a counter the radioactivity as a function of time, thus making arterial sampling superfluous. The theory predicts that the weighted mean of the concentration of indicator in any number of blood vessels can be measured as a function of time and used for calculating cardiac output in the same way as mentioned above (Burger ${ }^{1}$ and van der Feer ${ }^{2}$ ).

To enable separation of the primary part of the counting rate-time curve obtained in this way, there must be present a sufficiently high peak belonging to the indicator that passes for the first time. For a curve to be good this peak must be short and high. Extrapolation will be easy then, and relatively unimportant, because the area of the extrapolated part will be small in comparison with the area of the measured part.

For a certain injection site and a short injection the quality of the curve depends on the choice of the part of the body that is taken as the measuring volume. Two properties of this volume are important: first, the time interval

\footnotetext{
From the Department of Medical Physics, Physical Laboratory of the University of Utrecht. Netherlands (Director: Prof. Dr. H. C. Burger).

This investigation was made possible by financial support from the "Gezondheidsorganisatie T.N.O., Medisch-Physische Afdeling."

Received for publication June 4, 1958.
} 
in which the indicator arrives at the different inflows of the measuring volume, and, second, the time it takes the indicator to traverse the measuring volume. Both have to be small in comparison with the mean circulation time of the whole circulatory system.

The "traversal time" is determined mainly by the size of the measuring volume. This volume can be made small by restricting the sensitive solid angle of the counter by a lead collimator. The spread of arrival times can be made small only by suitable choice of the measuring site, because the site will determine which blood vessels are chosen as measuring volume.

The experiments described here aimed to determine which regions of the human body give suitable curves, and to check the obtained values for cardiac output by the classical sampling method. Blood was therefore sampled from the brachial or femoral artery and the radioactivity measured continuously.

For the indirect measurements, calibration of the counter sensitivity in the position in which it is used is necessary. This was obtained by measuring the counting rate when the concentration of indicator had become uniform in the whole circulation. Since this uniform concentration is reached about $5 \mathrm{~min}$ utes after the injection, the indicator used is not allowed to leave the circulation in any appreciable amount during this period. We therefore made use of human serum albumin, iodinated with $\mathrm{I}^{131}$, which leaves the blood plasma at a rate of 0.1 per cent per minute (Wasserman ${ }^{10}$ ).

Since the amount of indicator that can be given is limited by the radiation dose received by the patient, we made use of scintillation counters, which are much more sensitive than the simple Geiger counters. The largest dose is received by the blood of the patient. With the usual approximations (Mayneord ${ }^{6}$ ) this was calculated for an injection of $50 \mu \mathrm{c}$ of $\mathrm{I}^{1 * 1}$ (the amount used by us) to about 1 roentgen, from which 0.5 roentgen is received in the first week after the injection.

The blood sampled was led past the crystal of one of the counters, with a constant flow rate obtained by a motor-driven syringe. Cardiac output was then calculated from the direct and from the indirect counting rate-time curve obtained simultaneously with one and the same injection of radioactive indicator.

\section{EOUIPMENT}

The scintillation counters used were of conventional design. A Tl-activated sodium iodide crystal of $35 \mathrm{~mm}$. diameter and $25 \mathrm{~mm}$. thickness was cernented on top of a photomultiplier (E.M.I. type 6260). Crystal and multiplier were mounted together in a lead cylinder having an outer diameter of $130 \mathrm{~mm}$. and a wall thickness of $35 \mathrm{~mm}$. The top of this cylinder was closed by an aluminum sheet $(1 \mathrm{~mm}$.); the free end of the crystal rested against this sheet. On top of the lead cylinder a lead collimator, 20 or $35 \mathrm{~mm}$. thick, could be mounted, having an aperture of $35 \mathrm{~mm}$. and giving a half-value angle for $I^{131}$ radiation of $45^{\circ}$ and $25^{\circ}$, respectively.

Pulses from the photomultiplier were equalized and recorded on photographic paper running at a speed of $3 \mathrm{~cm}$. per second. From this record the counting rate-time curve, as well as the integral of this curve, could be easily deduced.

Continuous sampling was done through an arterial needle inserted into the brachial or femoral artery. This needle was connected to stainless steel tubing, $55 \mathrm{~cm}$. long and of $2 \mathrm{~mm}$. bore, by means of polyethylene tubing, $35 \mathrm{~cm}$. long and of $2 \mathrm{~mm}$. bore. The stainless steel tubing was wound two and a half turns around the sodium iodide crystal of one of the scintillation counters 
and the free end connected to a motor-driven syringe by means of another piece of polyethylene tubing. Steel tubing and crystal were completely shielded by $35 \mathrm{~mm}$. of lead. The all-metal syringe had a capacity of $50 \mathrm{~cm} .{ }^{3}$, and the plunger was driven with a velocity that resulted in a constant pumping specd of $0.8 \mathrm{~cm} .^{3}$ per sccond. Dimensions and flow rate were chosen so that the response time of the sampling device was about 2 seconds. This was measured by pumping water containing $I^{131}$ through the device and introducing a sudden change in $I^{131}$ concentration at the inflow of the arterial needle. For measuring the integral of the primary curve the value of the response time is not important at all. In order to enable extrapolation of the downsloping part of the first peak, however, the response time must not be too long.

The activity of whole blood samples was determined by filling up a small, flat-bottomed bottle to a mark on the neck of the bottle and inserting it into the aperture of the scintillation counter head. The same bottle (contents about $3 \mathrm{~cm}^{3}$ ) was then filled with a standard solution of $I^{131}$ of a known dilution factor, and the results obtained were compared. The filling of the bottle is not at all critical, owing to its shape, and $I^{131}$ concentrations could be measured with an accuracy better than 1 per cent. The blood samples were hemolyzed by cooling at $-10^{\circ} \mathrm{C}$. After rewarming, a homogeneous solution is obtained. This was done because red cells would otherwise accumulate at the bottom of the bottle, resulting in a too low counting rate as compared with a homogeneous distribution of red cells, and thus of the active plasma.

Normally, the measurements on patients were made with three scintillation counters. One was used for measuring the activity of the sampled blood, and the other two for indirect measurement of concentration-time curves.

\section{PROCEDURE}

The solution of iodinated human serum albumin was diluted with saline to a concentration of about $50 \mu \mathrm{c}$ per $\mathrm{cm} .{ }^{3}$. Two syringes were filled with $1 \mathrm{~cm}^{3}$ of this solution and weighed. One was used for the venous injection, and the other was diluted with $5 \mathrm{~L}$. of water for calibrating purposes. After they had been used, the empty syringes were reweighed, in order to know the exact amount injected into the patient and the calibrating solution.

The sampling device was flushed with a sterile isotonic solution of citrate of sodium (3.6 per cent), which appeared to prevent clotting during the measurement. The counters were then brought into position and the arterial needle was inserted. After inserting the injection needle into a cubital vein, the stylette of the arterial needle was removed and the sampling device connected. Next, the recording camera was switched on, the indicator injected in about 1 second, and the motor of the sampling device switched on.

At the end of the sampling period (about 1 minute) the sampling device was disconnected from the arterial needle and flushed again with the solution of citrate of sodium.

About 4 minutes after the injection, the recording camera was switched off, and a $5-\mathrm{cm}^{s}$ sample of blood was taken through the arterial needle. The concentration of $I^{131}$ in this sample was measured as described in Section 2. The sampling device was then filled with the standard dilution and its counting rate determined.

To avoid uptake of free $I^{131}$ by the thyroid gland, the patient was given $1 \mathrm{Gm}$. of potassium iodide before the measurement, and $0.5 \mathrm{Gm}$. daily for 3 weeks thereafter.

\section{CAlculation of Cardiac output}

Cardiac output is then calculated from the above-described measurements with the formula:

$$
F=\frac{M U_{0}}{c_{0} \int_{t=0}^{\infty} U(t) d t}
$$

in which $M$ is the amount of indicator injected, $U_{o}$ the counting rate obtained when the measuring 
volume is filled with the concentration $c_{a}$, and $\int_{i=0}^{\infty} \mathrm{U}(\mathrm{t}) \mathrm{dt}$ the integral of the primary counting
rate-time curve (Burger ${ }^{1}$ ).

If $M_{p}$ denotes the amount of indicator injected into the patient, $M_{a}$ the amount of indicator used for the standard solution, $U_{\mathrm{s}}$ the counting rate measured when the sampling device is filled with the standard dilution, the output in cubed centimeters per second calculated from the sampling curve is given by:

$$
F_{a r t}=\frac{M_{p} U_{p}}{\frac{M_{s}}{5,000} \cdot \frac{N_{p}}{N_{s}} \int_{t=0}^{\infty} U(t) d t}=\frac{5,000 U_{s}}{\int_{t=0}^{\infty} U(t) d t} \cdot \frac{M_{p}}{M_{s}}
$$

In this formula, $\mathrm{U}_{\mathrm{s}}$ is measured in counts per second, and the integral of the arterial counting rate-time curve in number of counts.

The output calculated from an indirectly measured concentration-time curve is given by:

$$
F_{i n, t}=\frac{M_{p} U_{p}}{\frac{M_{s}}{5,000} \cdot \frac{N_{p}}{N_{s}} \int_{t=0}^{\omega} U(t) d t}=\frac{5,000 U_{p}}{\int_{t=0}^{\infty} U(t) d t} \cdot \frac{M_{p}}{M_{s} N_{p}}
$$

where $U_{p}$ is the counting rate measured when a homogeneous concentration is reached in the patient, $N_{s}$ and $N_{p}$ are the counting rates measured for the small sampling bottle filled with standard solution and patient's blood, respectively.

The blood of the patient is given by:

$$
V_{p}=\frac{M_{p}}{M_{s}} \cdot \frac{N_{s}}{N_{p}} \cdot 5,000
$$

If this is substituted in (.3), the following simple expression is obtained for $F_{\text {ind }}$ :

$$
F_{\text {ind }}=\frac{V_{p} U_{p}}{\int_{i=0}^{\infty} U(t) d t}
$$

If the value obtained for $F_{\text {ind }}$ is to be checked by the value $F_{a r t}$ obtained by the classical sampling method, $F_{i n d} / F_{a r t}$ is of interest only. If the two counting rate-time curves $U(t)$ are measured simultaneously with one injection of radioartive material, it can be seen from formulae (2) and (3) that it is not necessary to know the amounts $M_{p}$ and $M_{8}$ injected into patient and standard, respectively.

If one indirect value is to be checked by another indirect value measured simultaneously, even the finally reached concentration in patient's blood (values of $N_{s}$ and $N_{p}$ ) need not be known.

\section{COMPARATIVE MEASUREMENTS ON PATIENTS}

It has been poiuted out that it is the choice of a measuring site that mainly determines whether a successful counting rate-time curve will be found. With the scintillation counter described in Section 2, using a collimator $35 \mathrm{~mm}$. in thickness, such a curve was obtained only if the counter head was directed toward the heart or toward the head of the patient. For all other parts of the trunk, as well as parts of an arm or leg, the measured curve showed a steady rise to a final value without showing a peak. Therefore, only head and heart curves were measured simultaneously with an arterial sampling curve. 
All patients were in the supine position during the measurements. The head curves were made with the counter axis in the horizontal position and the aperture of the collimator pressed against the ear. The $35-\mathrm{mm}$. collimator was used in order to restrict the measuring volume to the head alone. It is necessary to choose the right ear if the injection is made into the right arm, otherwise the counter can "see" the right shoulder and, therefore, the indicator going from the right arm to the right heart will contribute to the concentration-time curve. This is not allowed to happen, since the indicator must first pass the main branch before its concentration is measured.

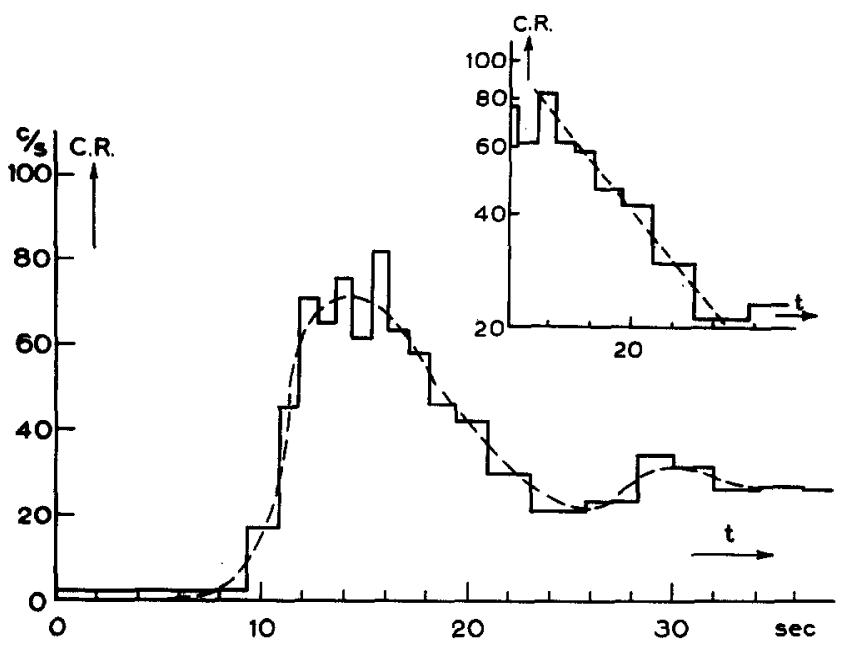

Fig. 1.-Typical counting rate-time curve obtained from the head counter. The downsloping part is plotted separately on a semilogarithmic scale.

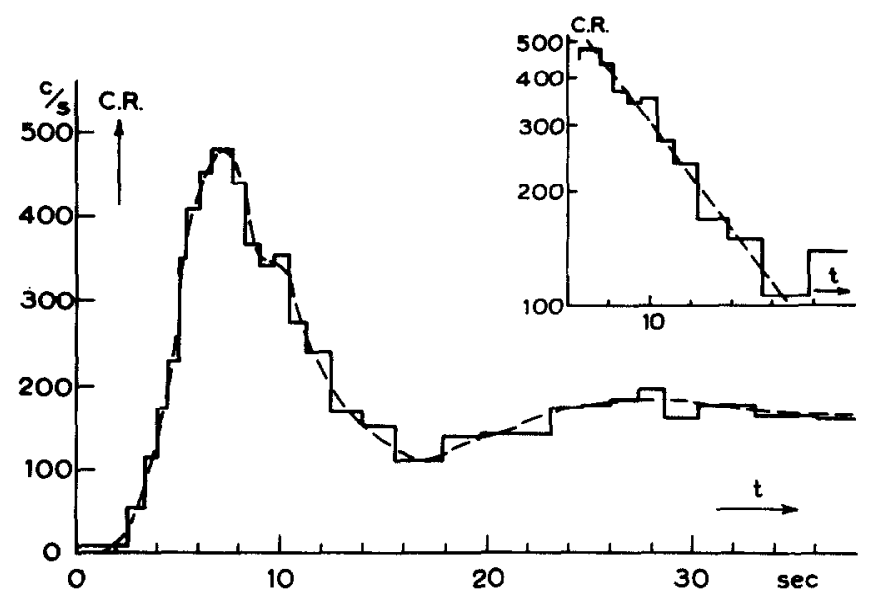

Fig. 2.-Typical heart curve measured on the same person as in Fig. 1, with the same injection.

The heart curves were measured with the counter axis in the vertical position and the aperture of the $20-\mathrm{mm}$. collimator above the center of the frontal heart projection, which was determined by percussion. The counter head was just free from the skin, in order to give the patient room for breathing. The $20-\mathrm{mm}$. collimator was used because the counting rates obtained with it were considerably higher than those with the $35-\mathrm{mm}$. one. 
Typical head, heart, and arterial sampling curves measured simultaneously in one patient with one injection of radioactive material are given in Figs. 1, 2, and 3, respectively.

If cardiac output is to be calculated from these curves, it is necessary to adopt some type of extrapolation of the downslope of the first peak. For arterial sampling curves the exponential extrapolation is a successful method for most cases. Since the downslope of the majority of our head and heart curves clearly showed an exponential part, we used the exponential extrapolation for all our curves.

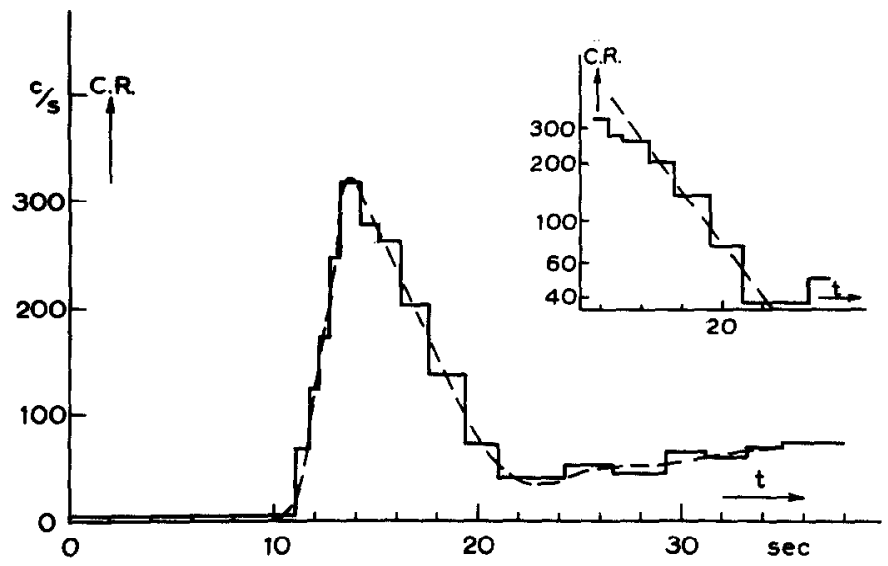

Fig. 3.-Typical arterial sampling curve measured on the same person as in Figs. 1 and 2, with the same injection.

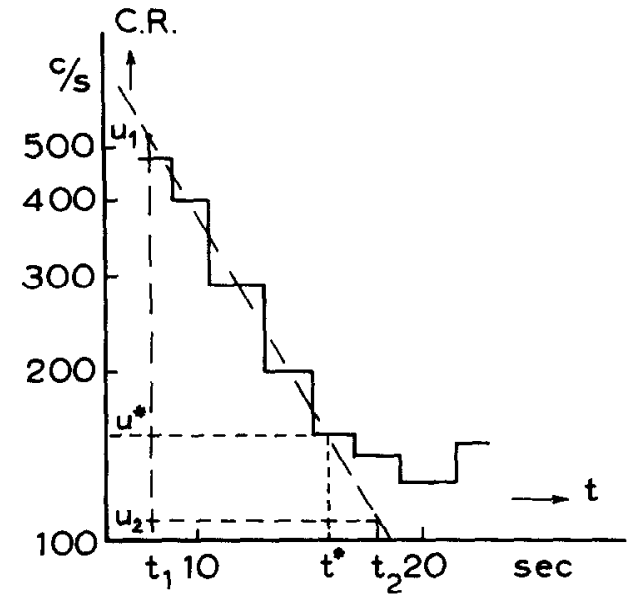

Fig. 4.-Plot of the downsloping part of a counting rate-time curve on a semilogarithmic scale.

The integral of the primary counting rate-time curve was determined by plotting the downsloping part on semilogarithmic paper. The integral of the measured part of this curve is obtained simply by counting on the record the number of pulses from time zero to $t^{*}$, where the measured curve leaves the straight line (Fig. 4). The number of counts obtained in this way must be diminished by the number of background counts over this same period.

If the exponential part is written as:

$$
U=U e^{\left.-\lambda_{(t}-t^{*}\right)}
$$


the integral of the extrapolated part is given by $U^{*} / \lambda$ where $U^{*}$ is the deflection at time $t^{*}$ and $\lambda$ is given by:

$$
\lambda=\frac{1}{t_{2}-t_{1}} \ln \frac{U_{1}}{U_{2}}
$$

where 1 and 2 are two arbitrary points on the straight line that is drawn through the measuring points (Fig. 4).

The results of the measurements in patients are presented in two scatter diagrams. In Fig. 5 the cardiac output values calculated from the head curves are compared with the values calculated from the arterial sampling curves. In Fig. 6, heart values are compared with arterial values.

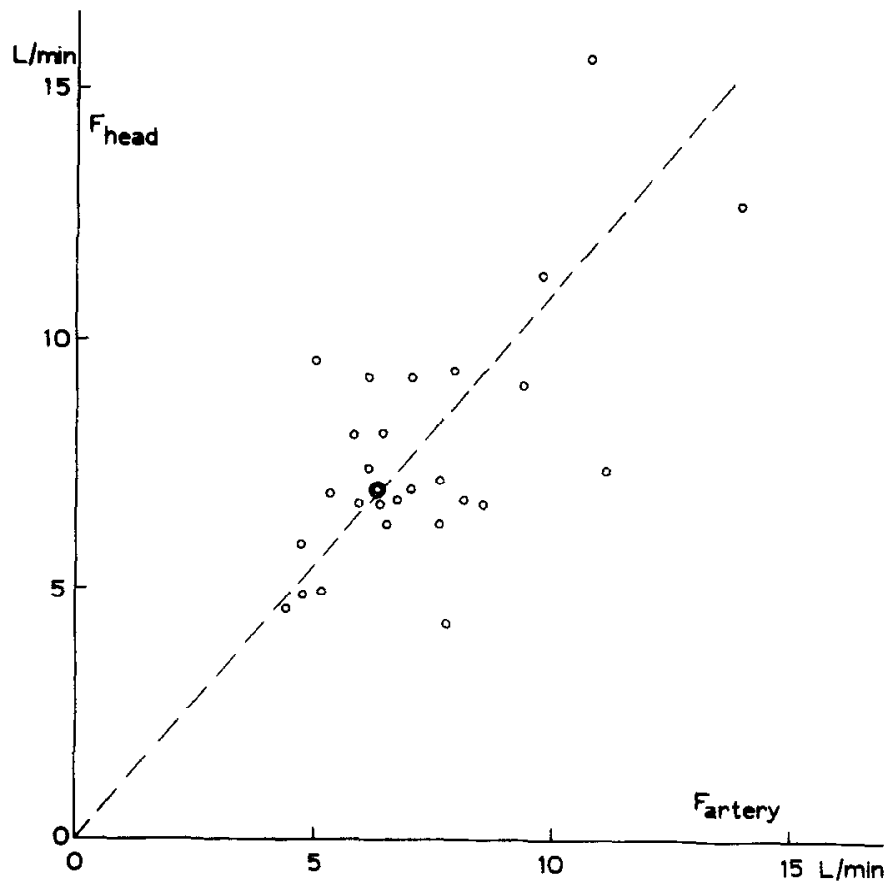

Fig. 5,-Comparison of cardlac output values calculated from a head curve and an arterial sampling curve in 29 cases. The two concentric circles represent two cases with identical values.

\section{DISCUSSION}

To get an idea of the validity of the cardiac output values calculated from an indirectly measured head or heart curve, it is assumed that the relation between arterial and head values (Fig. 5) and between arterial and heart values (Fig. 6) is given by a straight line going through the origin. So we make the fairly reasonable assumptions that the random errors of arterial, head, and heart values measured simultaneously are independent of one another, and that the random as well as the systematic errors are proportional to the value of cardiac output.

The mean value of $100\left(F_{\text {head }}-F_{\text {art }}\right) / F_{\text {art }}$ for all 29 measurements of Fig. 5 was calculated to +10 per cent, with a standard deviation of 5 per cent. The relation between $F_{\text {head }}$ and $F_{a r t}$ is, therefore, $F_{\text {head }}=1.10 F_{\text {art }}$ (dashed line in 
Fig. 5). The standard deviation of one comparison is 27 per cent. Since the standard deviation of $F_{\text {art }}$ is not more than 15 per cent (van der Feer ${ }^{2}$ ), the standard deviation of $F_{\text {head }}$ is more than 23 per cent $\left(23^{2}=27^{2}-15^{2}\right)$.

The mean value of $100\left(\mathrm{~F}_{\text {heart }}-\mathrm{F}_{\mathrm{art}}\right) / \mathrm{F}_{\mathrm{art}}$ for all 33 measurements of Fig. 6 was calculated to +13 per cent, with a standard deviation 2.5 per cent. Thus, $F_{\text {heart }}=1.13 \mathrm{~F}_{\text {art }}$. The standard deviation of one comparison is 15 per cent. This means that the standard deviation of $F_{\text {heart }}$ is of about the same size as the standard deviation of $F_{a r t}$, or it may be even smaller. Therefore, we also calculated the mean value of $100\left(F_{\text {art }}-F_{\text {heart }}\right) / F_{\text {heart }}$. This gives the relation $F_{\text {heart }}=1.11 F_{\text {art }}$ and a standard deviation of one comparison of 12 per cent.

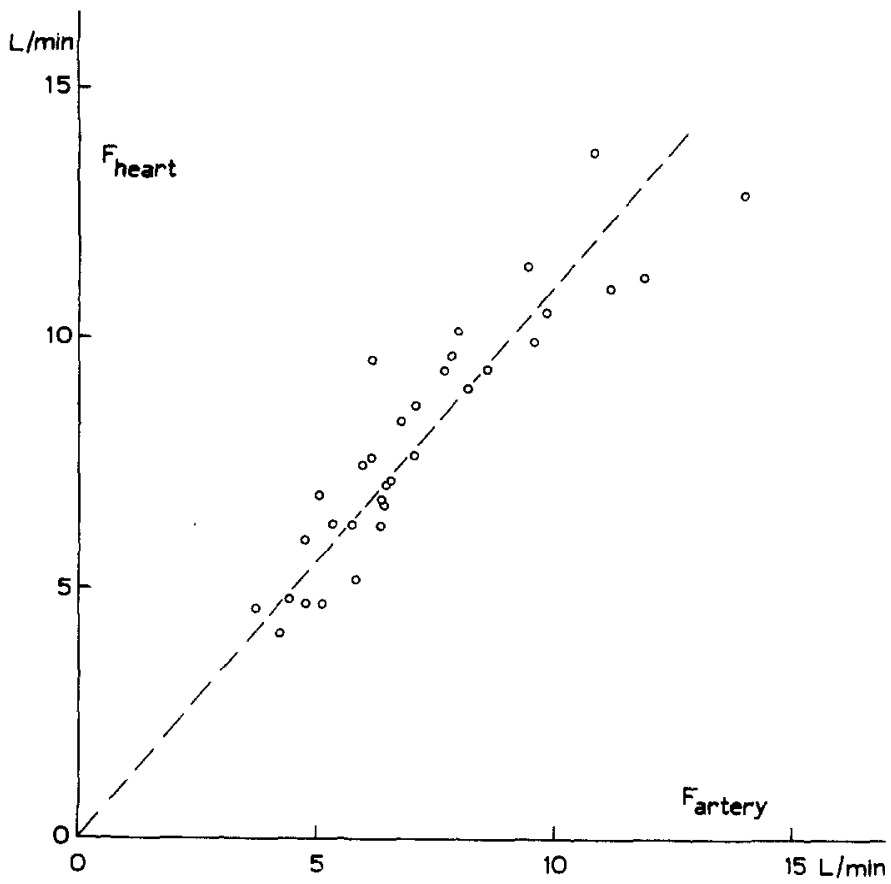

Fig. 6.-Comparison of cardiac output values calculated from a heart curve and an arterial sampling curve in 33 cases.

The dashed line in Fig. 6 represents the mean of the two relations, that is, $\mathrm{F}_{\text {heart }}=1.12 \mathrm{~F}_{\text {art }}$. The standard deviation of $\mathrm{F}_{\text {beart }}$ cannot be evaluated without knowing more about the standard deviation of $\mathrm{F}_{\mathrm{art}}$ in this particular case. If we assume, however, that both standard deviations are of about the same size, 10 per cent $\left(10^{2}+10^{2}=15^{2}\right)$ is a good approximation.

So our final conclusion is that an indirectly measured head curve could be used for calculating cardiac output, but it is not very successful because of the rather high standard deviation of the result, that is, about 25 per cent.

An indirectly measured heart curve, however, can replace successfully an arterial sampling curve. Because of the systematic error of +12 per cent, the cardiac output value calculated from a heart curve must be corrected with -11 per cent. The standard deviation of the result is about 10 per cent. 
These figures may be compared with the material presented by others. Huff $^{3}$ checked output values calculated from an indirectly measured heart curve against the direct Fick method, in dogs. Albumin iodinated with $\mathrm{I}^{131}$ was used, and the scintillation counter was well collimated with a $5-\mathrm{cm}$. collimator having an aperture of $2 \mathrm{~cm}$. in diameter. The curves were extrapolated exponentially. In 22 measurements no systematic error between the two methods was found. From his material we calculated a standard deviation of 21 per cent for one comparison.

Pritchard ${ }^{7}$ compared indirectly measured heart curves with arterial sampling curves in about the same way as we did. With a well-collimated scintillation counter, exponential extrapolation of the heart curves was possible, and a good agreement between the two output values was found. For 33 out of 39 measurements the difference was not more than 7 per cent. Unfortunately no more figures were given in this short abstract, and, therefore, no standard deviation could be calculated.

The same comparisons were made by $\mathrm{Mack}^{5}$ in 10 cases. The curves were also extrapolated exponentially. No systematic difference between heart and arterial values was found. We calculated a standard deviation of 13 per cent for one comparison.

Shackman ${ }^{4 a}$ made 61 comparisons of cardiac output values calculated from an indirectly measured heart curve with the direct Fick method, in man. The scintillation counter used had a collimator of $5-\mathrm{cm}$. thickness, with an aperture of $2.5-\mathrm{cm}$. diameter, which gives a half-value angle of $23^{\circ}\left(\right.$ Veall $\left.^{9}\right)$. The mean difference between the two methods is reported as $175 \mathrm{~cm} .{ }^{3}$ per minute, with an error of $134 \mathrm{~cm}^{3}$ per minute. If we assume that the mean value of cardiac output is $6 \mathrm{~L}$. per minute, this means that the systematic error is $3 \pm 2$ per cent and the standard deviation of one comparison about 15 per cent.

The figures mentioned above are all in good agreement with our own findings, except for the systematic error of 12 per cent. This error is due probably to the solid angle of our heart counter, which is rather wide (half-value angle of $45^{\circ}$ ). Thereby the calibrating deflection of this counter may be too high because the counter is capable of observing too many blood vessels which do not contribute to the first peak of the concentration-time curve. Therefore, the use of a collimator that gives a half-value angle of not more than $25^{\circ}$ is recommended. This necessitates, however, directing the counter toward the heart accurately.

The presence of a systematic error in our heart and head values actually means that the type of extrapolation used is not the right one. That the exponential extrapolation can be used for a heart curve measured with a highly collimated counter can be understood as follows. The heart counter, sufficiently collimated, chiefly measures the concentration of indicator in the heart. The downslope of the primary part is mainly determined by the concentration of the left heart. Since the shape of the concentration-time curve of the left heart is not very different from the shape of the concentration-time curve in the big arteries, it is not surprising that the exponential extrapolation is so successful. The downslope of a head curve, however, consists of contributions from the 
whole head and is exponential probably only in the first approximation. Thus, a systematic error is introduced by using the exponential extrapolation.

The great difference in standard deviation of heart and head values probably may be attributed also to the extrapolation, since the slope of the straight line drawn on semilogarithmic paper is less accurate in the case of a head curve because of the lower counting rate.

\section{SUMMARY}

Cardiac output was measured by the injection method, using $\mathrm{I}^{13 \mathrm{I}}$-labeled human serum albumin as indicator, and measuring concentration-time curves through the intact skin with a collimated scintillation counter. Although, in principle, any number of blood vessels could be used as the measuring site, it was found that because of recirculation, suitable curves were obtained only if the counter was directed toward the head or the heart. Cardiac output values calculated from these curves were compared with values found with the classical sampling method. Head, heart, and arterial sampling curves were measured simultaneously with one injection of radioactive indicator.

The measurements show that a head curve could be used in measuring cardiac output by the injection method, but it is not very successful because of the rather high standard deviation of the result (25 per cent). A heart curve, however, can successfully replace an arterial sampling curve. The accuracy of heart and arterial values is about the same (standard deviation 10 per cent). The systematic error of +12 per cent found for our heart values might be reduced by using a collimator that gives the counter a half-value angle of not more than $25^{\circ}$.

\section{REFERENCES}

1. Burger, H. C., van der Feer, Y., and Douma, J. H.: Acta cardiol. 11:1, 1956.

2. van der Feer, Y.: Thesis, Utrecht, 1958 (English text available on request from the author).

3. Huff, R. L., Feller, D. D., Judd, O. J., and Bogardus, G. M.: Circulation Res. 3:564, 1955.

4. Lammerant, J.: Le volume sanguin des poumons chez l'homme, Bruxelles, 1957, Editions Arscia.

4a. Shackman: See Lammerant. ${ }^{4}$

5. Mack, R. E., Wells, H. J., and Pollack, R.: Radiology 68:245, 1957.

6. Mayneord, W. V.: Brit. J. Radiol., Suppl. 2, 1950.

7. Pritchard, W. H., MacIntyre, W. J., and Moir, T. W.: J. Lab. \& Clin. Med. (Abstract) $46: 939,1955$.

8. Stewart, G. N.: Am. J. Physiol. 57:27, 1921.

9. Veall, N., Pearson, J. D., Hanley, T., and Low, A. E.: Proceedings, Second Radioisotope Conference, Oxford 1:183, 1954.

10. Wasserman, K., and Mayerson, H. S.: Am. J. Physiol. 165:15, 1951. 